\title{
Laser-Doppler measurements on a round turbulent jet in dilute polymer solutions
}

\author{
BY STEVEN J. BARKER \\ Graduate Aeronautical Laboratories, California Institute of Technology, Pasadena
}

(Received 11 August 1972 and in revised form 11 April 1973)

A laser-Doppler velocimeter suitable for the measurement of mean and fluctuating flow velocities in water is described. Results of a study using this system in an axisymmetric turbulent jet of water and dilute polymer solutions are given. The laser-Doppler technique is better suited for such measurements than either Pitot tubes or heat-transfer gauges because the Doppler velocity measurements are independent of the physical properties of the fluid. Previous velocity measurements in polymer jets have suffered from the effects of the additives upon the sensors.

Turbulent round jets with Reynolds numbers between 5000 and 50000 were studied. For a jet issuing from a convergent nozzle the additives were found to have no effect upon the mean axial velocity or turbulence intensity at any point in the jet. However, for a jet issuing from a long length of circular pipe, the additives reduced the centre-line velocity and increased the turbulence level in the early part of the jet. Thus the principal effect of a polymer additive upon the jet appears to result from its effect upon the initial conditions.

\section{Introduction}

\subsection{Polymer flows}

The reduction of turbulent skin friction by polymer additives of high molecular weight has been known for twenty-five years, yet it is not well understood today. The phenomenon has been characterized in many experiments, such as those described in the Proceedings of the 1968 Symposium on Drag Reduction (Wells 1969). Most of the drag-reduction mechanisms that have been proposed rely upon a polymer effect on small-scale turbulence in the viscous sublayer or buffer zone of the boundary layer. Opinions vary as to the nature of this effect and what polymer property is responsible. Lumley (1969) has stated that the solution viscoelasticity, in particular the high resistance to axisymmetric strain fields, is essential to the drag-reduction process. However, Brennen \& Gadd (1967) found that elastic effects in a dilute solution of polyethylene oxide ('Polyox') disappear after the solution has been aged for several days, but the aged solution is still a very effective drag reducer.

Other authors have proposed particulate polymer effects. Van Driest (1967) supposes a 'lattice of interconnected long-chain polymers' which suppress turbulent eddies near the wall by purely viscous interaction. Although the 
polymer molecules themselves are much smaller than the dissipative turbulent eddies, they are known to have the ability to form agglomerations (Fabula 1966). Whether such agglomerations or a continuum property of the solutions cause the drag reduction is as yet unknown.

A way to verify that only the small-scale turbulence is affected by additives is to study free turbulent flows of dilute polymer solutions. If inertial-scale eddies are uninfluenced by polymers, then turbulent flows in the absence of a wall should be little affected. Fabula (1966) has studied grid turbulence in polymer solutions using hot-film anemometers. He found that polymer solutions produced 'ragged ' hot-film signals, apparently owing to effects of molecular agglomerations. For solutions which gave ragged signals, no results could be obtained. For solutions of Polyox this raggedness would decay with ageing and no effect upon the turbulence spectrum was found in the aged solutions. Greated (1969) has also studied grid turbulence in polymer solutions, using a laser-Doppler velocimeter. He found that the polymer Separan AP-30 caused a slight decrease in the turbulence level and a steepening of the turbulent spectrum at the high frequencies.

Submerged jets provide another means of studying turbulent flow in the absence of a wall. A simple visual jet study by Gadd(1965) suggested that smallscale turbulence may be suppressed by a 30 parts per million (p.p.m.) solution of Polyox. Another visual study by A. White (1969) showed that solutions of guar gum at concentrations up to 500 p.p.m. had no visible effect upon the round jet. However, solutions of Polyox exhibited apparent turbulence damping at concentrations above 10 p.p.m., with results similar to those of Gadd. White also found that, when the Polyox solutions were aged for a few days, the turbulence suppression disappeared.

These flow visualization studies suggest that quantitative measurements in polymer jets are needed. Past attempts to make such measurements have suffered from the anomalous effects of the additives upon the velocity probes. Hot-film anemometers and Pitot tubes do not measure flow velocity directly. Both give results which are dependent upon some of the physical properties of the fluid. Both techniques are adversely affected by the non-Newtonian properties of polymer solutions, as was found by Friehe \& Schwartz (1969). Thus it is not surprising that contradictory results have been obtained using these methods. Jackley (1966) studied a polymer jet using Pitot tubes and found that the additives had no measurable effect. In a similar experiment, D. A. White (1967) found that a jet of Polyox had a significantly greater spreading angle than a pure water jet.

There are three possible reasons for the difference in the results of these two experiments. One is Pitot tube error caused by the polymer additives. Another is differences in polymer solution preparation techniques. As noted above (A. White 1969), the age of a solution may affect its behaviour in a submerged jet. The third possible cause is the effect of the polymer upon the initial conditions of the jet. Since the study of D. White extended only 25 jet orifice diameters downstream, such initial perturbations would not necessarily have decayed away. 


\subsection{Laser-Doppler velocimetry}

In an attempt to resolve the discrepancies of the results cited above, a submergedjet experiment has been performed using a different technique to measure velocity. The laser-Doppler velocimeter $(\mathrm{LDV})$ is a device that provides a direct measurement of velocity which is independent of the physical properties of the fluid. The results of LDV measurements are not affected by the non-Newtonian properties of dilute polymer solutions.

The basic principle of the LDV is as follows. Laser light which has been scattered by small particles moving with the fluid flow has a Doppler frequency shift owing to the motion of the scattering particles. This frequency shift is measured by heterodyning the scattered, Doppler-shifted light with unscattered light from the same laser. The heterodyning is done optically by combining the scattered and unscattered light on the surface of a photodetector. The detector then produces a beat frequency related to the particle velocity by

$$
f_{d}=(n / \lambda) \mathbf{u} \cdot\left(\mathbf{k}_{s e}-\mathbf{k}_{i}\right)
$$

where $f_{d}$ is the beat frequency, $n$ is the index of refraction of the fluid, $\lambda$ is the vacuum wavelength of the laser light, $\mathbf{u}$ is the particle velocity vector and $\mathbf{k}_{i}$ and $\mathbf{k}_{s c}$ are unit vectors in the directions of the incident and scattered light respectively. Thus the frequency $f_{d}$ is directly proportional to a single component of the particle velocity, and provides a measurement of that component.

Two different scattering geometries are in common use today. In the 'local oscillator' LDV mode, two coherent laser beams of different intensities are crossed at some point in the flow. The photodetector aperture is aligned with the weaker beam after it has passed through the flow. The detector sees direct light from one beam (the 'reference beam') mixed with scattered, Doppler-shifted light from the other beam. This method was first successfully used by Yeh \& Cummings (1964). The second LDV geometry is the 'dual scatter' mode, first proposed by Rudd (1969). Here two laser beams of equal intensity are crossed in the fluid, and in this case scattered light from both beams is gathered and mixed in the photodetector. This method has the advantage that more scattered light can be focused on the detector than in the local oscillator mode. On the other hand, the local oscillator mode usually produces less low-frequency noise due to random beam amplitude modulation. Using either scattering geometry the LDV has the advantages of linearity, small probe volume, no interference with the fluid flow and independence of the fluid rheology.

For a given optical arrangement, there are three basic methods of processing the signal from the LDV photodetector. The simplest of these is to measure the frequency spectrum of the Doppler signal itself with a wave analyser (Yeh \& Cummings 1964). Then the peak in the Doppler spectrum corresponds to the mean velocity and the width of the spectral peak is related to the turbulence intensity. The second method is that of 'individual realizations', whereby the frequencies of signal bursts from individual scattering particles are measured and used to construct a velocity histogram (Donohue, McLaughlin \& Tiederman 1972). Such histograms can be used to find the turbulence levels but do not give 


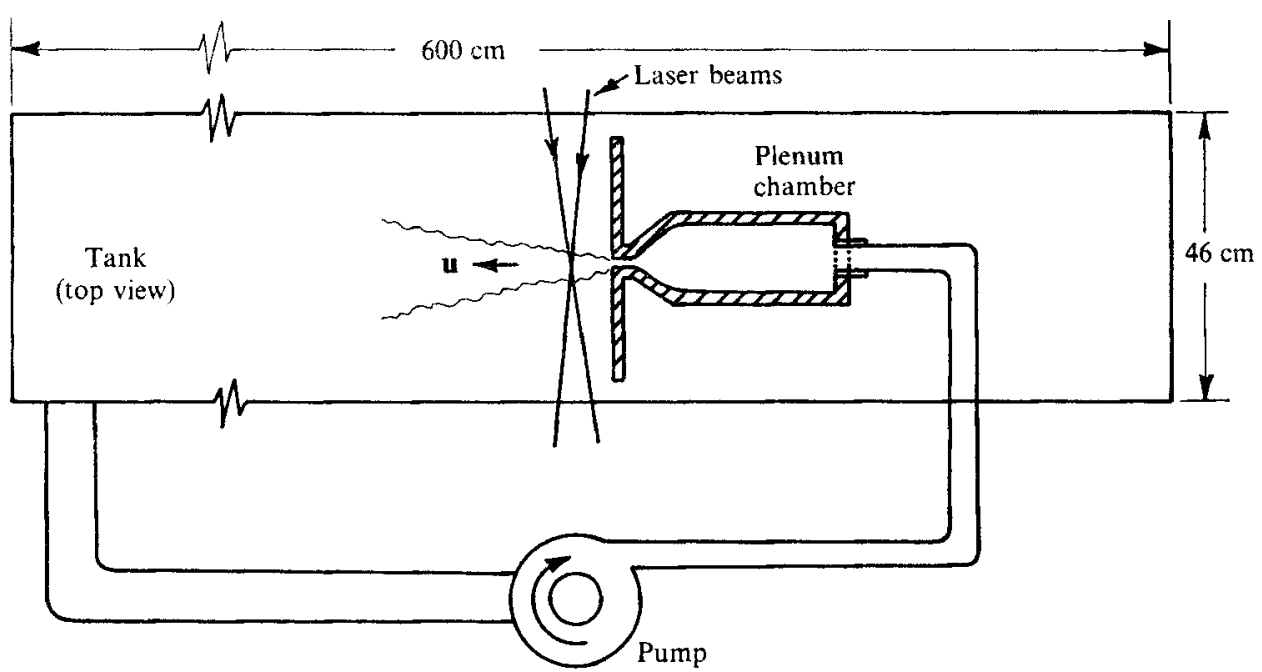

Figure 1. Experimental configuration.

the instantaneous velocity continuously. The third method is that of continuous frequency demodulation, in which the Doppler frequency is converted to an analog voltage which is then proportional to the instantaneous velocity. To make this possible there must be a high enough density of scattering particles to produce a nearly continuous Doppler signal, with very rare 'drop-outs'.

The accuracy of a continuous demodulation scheme is limited by the so-called 'ambiguity broadening' (George 1972). This broadening of the Doppler spectrum shows up as extraneous noise on the demodulated signal and has three causes: (i) gradients of mean velocity across the measuring volume of the LDV, (ii) turbulent fluctuations within the measuring volume and (iii) the finite time of transit for particles going through the measuring volume. The relative importance of these three factors in the present experiment will be discussed later. Although the ambiguity noise limits the measurement of small velocity fluctuations, it has no effect upon the mean velocity measurements.

Further analytical work on LDV signal processing can be found in Adrian (1972) and Wang (1972). Examples of signal-processing electronics are described in Stevenson \& Thompson (1972).

\section{Experimental apparatus}

\subsection{Round turbulent jet}

The axisymmetric jet used in this study is submerged in a glass-walled tank whose dimensions are $46 \times 46 \times 600 \mathrm{~cm}$ (figure 1). Fluid is pumped through the closed circuit by a centrifugal pump which is run at very low speed to avoid shear degradation of the polymer solutions. Before leaving the jet orifice, the fluid passes through a plenum chamber and a convergent nozzle whose area ratio is 30:1. Two different jet orifice diameters are used in the experiment: $0 \cdot 64$ and $1.91 \mathrm{~cm}$. 


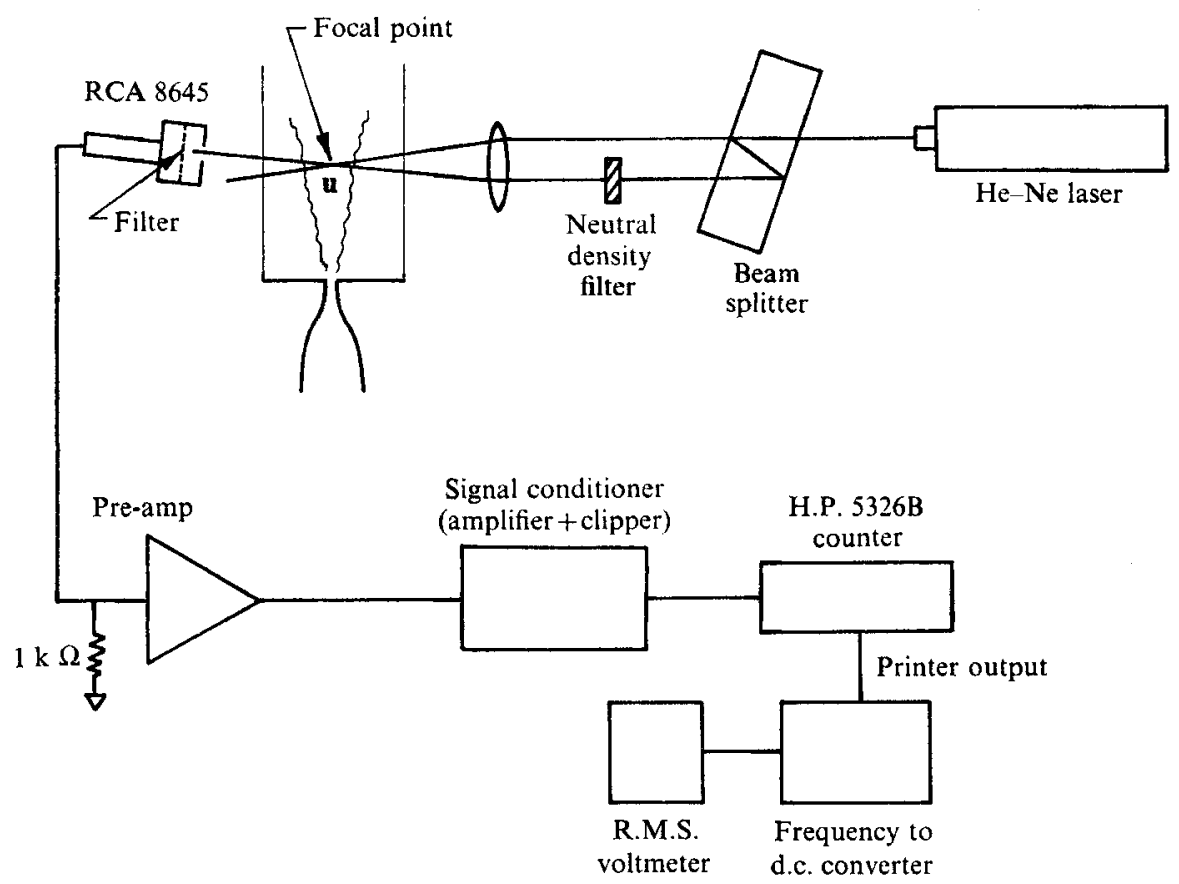

Figure 2. LDV block diagram.

Owing to the large volume of the experimental tank $\left(2.5 \times 10^{6} \mathrm{ml}\right)$, the time required for all of the fluid to circulate through the pump is of the order of $1 \mathrm{~h}$. Therefore, no measurable polymer shear degradation is expected during the time of the experiment. To verify this, solution samples were taken before and after each run and put through a simple pipe flow apparatus which qualitatively measured the drag-reduction effectiveness of the solutions. No shear degradation was observed during the experiments.

The polymer additive used was polyethylene oxide, sold by Union Carbide under the trade name Polyox, grade WSR-301. This is a linear chain polymer with an average molecular weight of several million. It is a very effective drag reducer in concentrations from 20 to 200 p.p.m. Each test solution was prepared by first mixing a master solution in a 60 gallon tank. The master solution was aged for $24 \mathrm{~h}$ and then was pumped into the experimental tank. The final solution in the tank was gently mixed for $2 \mathrm{~h}$ before the experiment was run. This procedure produced repeatable time-independent results.

\section{2. $L D V$ instrumentation}

The laser-Doppler velocimeter used in this experiment operates in the local oscillator mode as described in $\$ 1.2$. The beam from a $5 \mathrm{~mW}$ helium-neon laser (Spectra Physics model 120) is split into two parallel beams by a glass-block beam splitter using partial internal reflexion (figure 2). The weaker beam from the splitter is then further attenuated by a number 2 neutral density filter, which reduces the intensity by $10^{-2}$. Both beams then pass through a single biconvex 
lens of focal length $25 \mathrm{~cm}$. Since the two beams are parallel and collimated when they reach the lens, they will be focused to a point at the location at which they cross, thus minimizing the focal volume. The principal advantage of this system over those which use separate optical components for each beam is that it requires no alignment. Simple calculations estimate the size of the focal volume as $1 \times 0.05 \times 0.05 \mathrm{~mm}$.

After the two beams have passed through the tank, the weaker reference beam passes through a $1 \mathrm{~mm}$ aperture and an optical filter, and then enters an RCA model 8645 photomultiplier tube. Here the reference beam is mixed with scattered light from the brighter beam, producing the Doppler signal. This signal is amplified by $40 \mathrm{db}$ and then band-pass filtered to remove frequencies outside the range of interest. The Doppler signal is then further amplified and clipped by Zener diodes to remove most of the random amplitude modulation of the raw photomultiplier signal. The resulting signal, which is roughly a square wave, is fed directly into a digital frequency counter which averages the Doppler frequency over periods of up to $10 \mathrm{~s}$.

If there are frequency drop-outs of the Doppler signal owing to insufficient scattering particles in the focal volume, the frequency read by the counter will be in error. This error can be determined by measuring the spectrum of the Doppler signal with a wave analyser and comparing the frequency at which the Doppler spectrum is peaked with the digital counter reading. In the present experiment the difference between these two frequencies was less than $2 \%$. This result was obtained using tap water with no additional scattering particles added; the ambient impurities in the water were sufficient.

To frequency demodulate the Doppler signal and measure velocity fluctuations, the digital counter is switched to the manual or free running mode and used as a pulse shaper and frequency divider. While the counter is running, the digital data output on the back produces a continuous square wave whose frequency is a fraction of the Doppler frequency. The value of this fraction depends upon which pin of the output plug is chosen. Frequency division by ten is used in this experiment. The resulting square wave is then fed into an Anadex model PI-408R Frequency-to-Voltage Converter. This device produces an analog voltage proportional to the Doppler frequency and thus proportional to the velocity. The frequency division and the characteristics of the converter have the effect of averaging the frequency over about 50 periods, which reduces the effects of signal drop-outs and high-frequency ambiguity noise. A typical Doppler frequency in the experiment is $300 \mathrm{kHz}$, so that the demodulated velocity signal has a bandwidth of $6 \mathrm{kHz}$. This velocity signal can then be fed into an r.m.s. voltmeter or spectrum analyser to measure the turbulence intensity or velocity spectrum.

The chief difference between this method of demodulation and most others in use, including that of DISA (Schmid 1972), is that the present system does not employ a frequency tracker or phase-locked loop. The filtered, clipped Doppler signal is fed directly into the frequency divider (digital counter) and frequencyto-voltage converter. The reason for this is that frequency-tracking or phaselocked electronics can follow rapid changes in the signal frequency only over a 
finite range. For the high turbulence levels encountered in the round jet, typically $20 \%$ or more, a phase-locked loop will not track the larger fluctuations in frequency accurately. An LDV system using the phase-lock technique has also been used at Caltech, but only in flows where the turbulence level is less than $12 \%$. At very low turbulence levels, the phase-lock method has the advantage of further reducing the effects of Doppler signal drop-outs.

As stated in $\$ 1.2$, the accuracy of the demodulation is limited by the Doppler ambiguity noise, which has three different components. In the case of the present experiment, the ambiguity noise due to mean velocity gradients or turbulent fluctuations within the focal volume is negligible, except in the thin shear layer very near the jet orifice. The remaining ambiguity noise, which is caused by the finite particle transit time through the focal volume, will be the same in laminar as in turbulent flow for a given mean velocity. The noise can then be determined at least approximately by measuring the fluctuations in the demodulated signal for the nearly laminar flow at the orifice of the jet. This measurement shows that ambiguity noise is equivalent to a turbulence level of $0.25 \%$, which becomes the resolution limit of the system. As stated by George (1972), the ambiguity noise will most seriously affect the high-frequency part of the measured velocity spectrum. For this reason velocity fluctuation spectra are not presented in this paper.

\section{Results}

Three types of measurements were made in the submerged jet. The axial component of the mean velocity was measured as a function of distance downstream $(x)$ and distance from the central axis of the jet $(y)$. Each mean velocity measurement is an average over a $30 \mathrm{~s}$ period. The turbulence level $u^{\prime} / U_{m}$, where $u^{\prime}$ is the r.m.s. value of the velocity fluctuations and $U_{m}$ is the local centre-line mean velocity, was measured at the same locations as the mean velocity. In addition, the frequency spectrum of the Doppler signal was measured at several locations to provide a check upon the digital counter and demodulator results (see $\S 1 \cdot 2$ ).

For each of the two jet orifice diameters, 0.64 and $1.91 \mathrm{~cm}$, three different initial velocities were used, producing a range of jet Reynolds numbers $R e=\rho U_{0} d / \mu$ from 5000 to 50000 . For each set of initial conditions, the mean velocity profile $U(y)$ was measured at four or more values of $x$. Each measurement made in pure water was duplicated exactly in the polymer solutions.

Figure 3 shows the mean velocity profiles of the early part of the jet in normalized form for water and for a 50 p.p.m. solution of Polyox. Here $y_{\frac{1}{2}}$ is the profile half-width, or the value of $y$ for which $U=\frac{1}{2} U_{m}$, and $d$ is the orifice diameter. For values of $x / d$ equal to or greater than $5 \cdot 3$ the data points fall nearly on the same curve. The polymer additive has no effect upon the velocity profiles either in the normalized form shown here or in their dimensional form. Figure 4 is a plot of the inverse centre-line velocity $U_{0} / U_{m}$ versus the downstream distance $x / d$, again showing no measurable effect by the additives. Measurements were also made using 100 p.p.m. solutions of Polyox with the same result.

The turbulence level at the jet centre-line is plotted as a function of $x / d$ in figure 5. The growth of the centre-line turbulence level to a value of about $0 \cdot 2$ is 


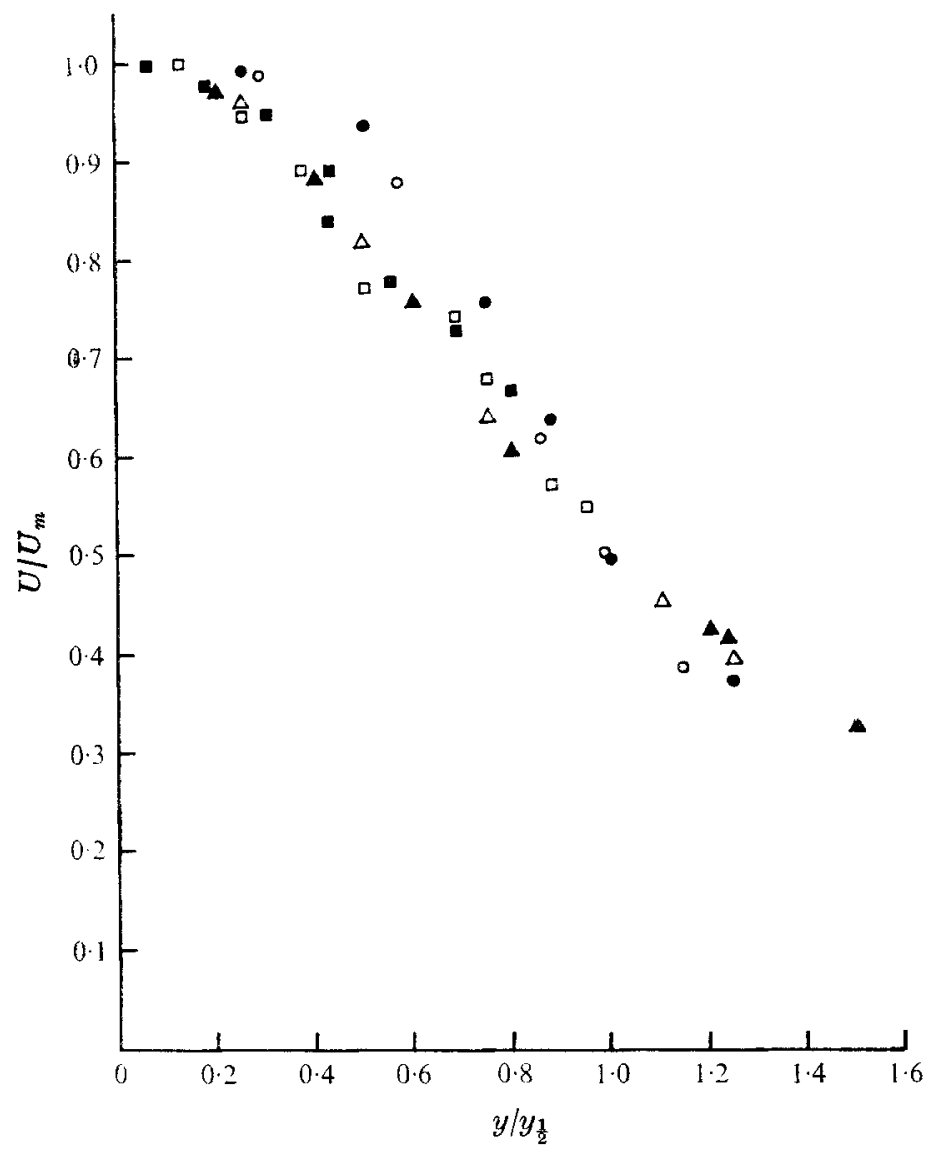

Figure 3. Mean velocity profiles. Open symbols, water; filled symbols, Polyox. $\bigcirc, x / d=2 \cdot 6 ; \Delta, x / d=5 \cdot 3 ; \square, x / d=10 \cdot 5$.

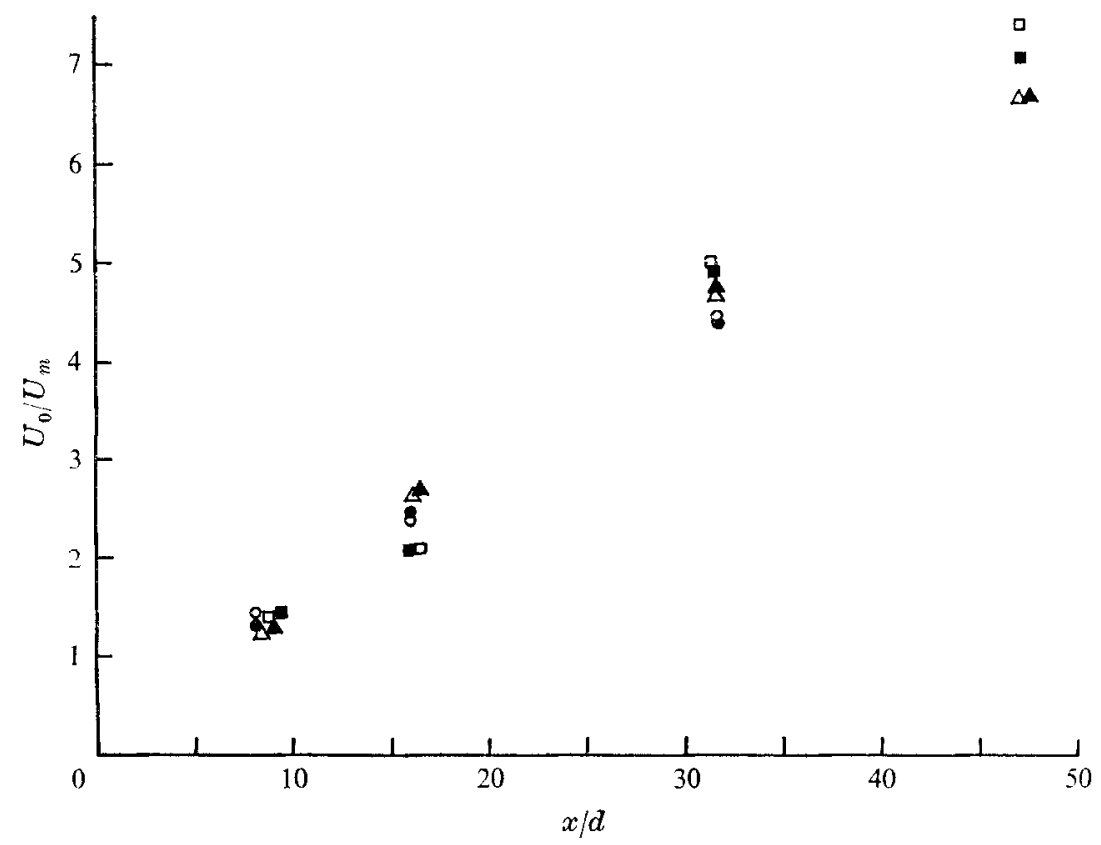

Figure 4. Centre-line velocity vs. $x / d$. Open symbols, water; filled symbols, Polyox. $\bigcirc, U_{0}=122 \mathrm{~cm} / \mathrm{s} ; \Delta, U_{0}=257 \mathrm{~cm} / \mathrm{s} ; \square, U_{0}=340 \mathrm{~cm} / \mathrm{s}$. 


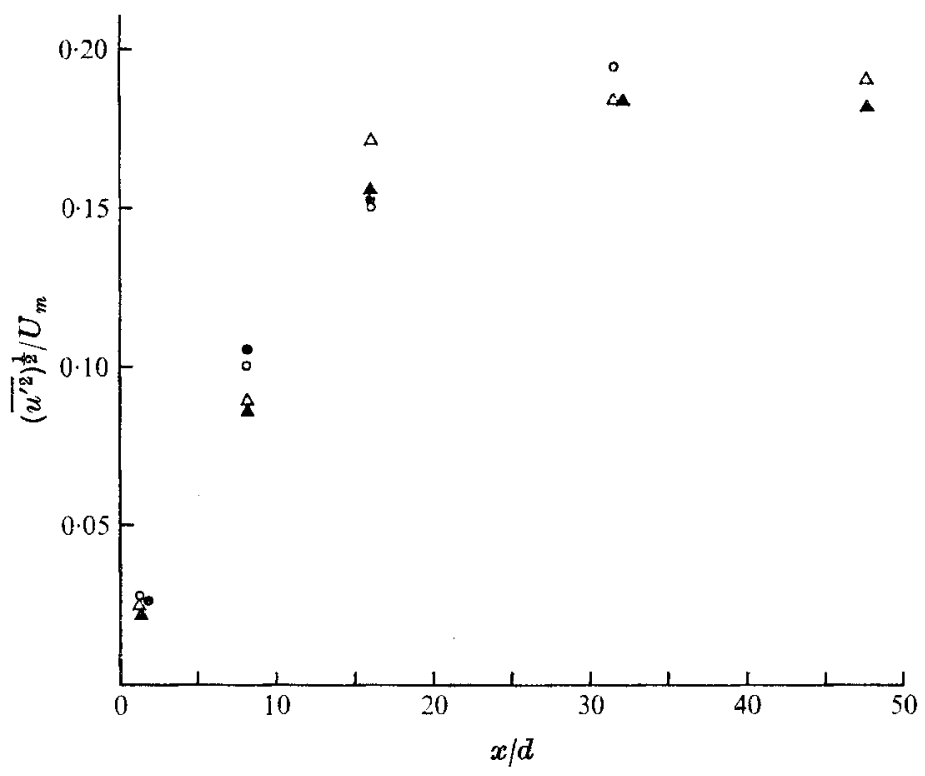

Frgure 5. Turbulence level $v s . x / d$. Symbols as in figure 4 .

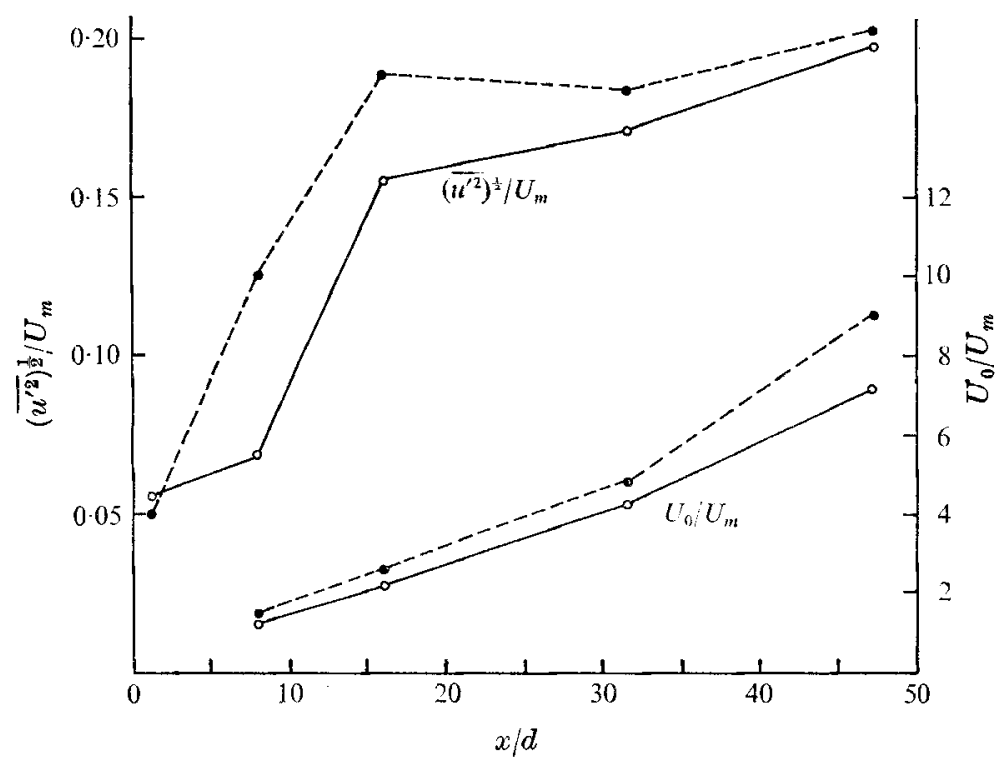

Figure 6. Results for jet from $62 \mathrm{~cm}$ tube. $\bigcirc$, water; $\bullet$ Polyox.

similar to previous results for round jets in air (Corrsin \& Uberoi 1949). Again the polymer additive is seen to have no measurable effect upon the jet.

In the measurements described above, the initial velocity profile of the jet at the orifice was nearly uniform except for a very thin boundary layer. The turbulence level at the orifice was below the resolution limit of the LDV system. Since no polymer effect was observed in this case, a different set of initial conditions was created. A tube of length $63 \mathrm{~cm}$ and inside diameter $0.63 \mathrm{~cm}$ was 
attached to the jet nozzle. The flow at the end of this tube is a fully developed turbulent pipe flow with a Reynolds number of 50000 . The jet emerging from the end of this tube was studied in the way described above.

Mean velocity and turbulence level results for this jet configuration are shown in figure 6 . The polymer additive reduces the centre-line velocity and increases the turbulence level during the early growth of the jet. The centre-line turbulence level of the flow as it emerges from the end of the tube is about 0.05 for both pure water and the polymer solution, but immediately downstream the level is higher in the polymer solution. The velocity profile in the polymer jet also has a greater spreading angle than in the water jet. A similar result was reported by D. A. White (1967) in his Pitot tube study.

It should be noted that the water and polymer jets being compared here are those having the same centre-line mean velocity at the end of the tube. Since the tube diameter is $0.63 \mathrm{~cm}$, it is impossible to measure the initial mean velocity profile in great detail. However, past measurements in pipe flows (Spangler 1969) indicate that the chief effect of the polymer upon the mean velocity is the thickening of the viscous sublayer and that the most significant effect upon the turbulence intensity occurs very near the wall. These differences apparently have an effect upon the early development of the jet, since no polymer effect was observed when the initial velocity profiles were uniform.

\section{Conclusions}

The results of this experiment show that inertial-scale turbulence in a round jet is not affected by polymer additives of high molecular weight, at least not when the solutions are prepared in the way described above. The only effect observed in the jet appears to result from the polymer's influence upon the initial flow conditions. The dependence of the downstream flow upon initial conditions may be the cause of some of the inconsistencies of previous experimental results. Effects of initial conditions are seen to persist downstream for 50 diameters or more.

The laser-Doppler technique has a significant advantage in flows of nonNewtonian fluids. The fact that the accuracy of the measurement is independent of the rheological properties of the fluid makes possible valid comparisons between polymer-solution and pure-solvent flows. Thus the results of this study cannot be questioned on the basis of polymer-induced instrument error. Some previous measurements using hot films or Pitot tubes are subject to doubt because of such errors.

\section{REFERENCES}

Adrian, R.J. 1972 J. Phys. E, Sci. Instrum. 5, 91.

Brennen, C. \& Gadd, G. E. 1967 Nature, 215, 1368.

Corrsin, S. \& Uberor, M. S. 1949 N.A.C.A. Tech. Note, no. 1865.

Donohue, G. L., Mclaughrin, D. K. \& Trederman, W. G. 1972 Phys. Fluids, 15, 1920.

FabULA, A. G. 1966 An experimental study of grid turbulence in dilute high-polymer solutions. Ph.D. thesis, Pennsylvania State University. 
Friehe, C. \& Schwartz, W. 1969 In Viscous Drag Reduction (ed. C. S. Wells), pp. 281296. Plenum Press.

GADD, G. E. 1965 Nature, 206, 463.

GeORge, W. K. 1972 In Proc. DISA Conf. on Flow in Industrial and Medical Environ. ments (ed. D. Cockrell), pp. 88-99. Leicester University Press.

Greated, C. A. 1969 Nature, 224, 1196.

JACKLEY, D. N. 1966 Drag reducing fluids in a free turbulent jet. U.S. Naval Ordnance Test Station, NAVWEPS Rep. no. 9053.

Lumley, J. L. 1969 Ann. Rev. Fluid Mech. 1, 367.

RuDd, M. J. 1969 Nature, 224, 587.

Sснмі, R. W. 1972 In The Use of the Laser-Doppler Velocimeter for Flow Measurements (ed. W. Stevenson \& H. Thompson), pp. 101-122. Office of Naval Research: Project Squid, Purdue University.

Spangler, J. G. 1969 In Viscous Drag Reduction (ed. C. S. Wells), pp. 131-157. Plenum Press.

Stevenson, W. H. \& Thompson, H. D. (ed.) 1972 The Use of the Laser-Doppler Velocimeter for Flow Measurements. Office of Naval Research: Project Squid, Purdue University.

VAN Driest, E. R. 1967 The damping of turbulent flow by long-chain molecules. Air Force Office Sci. Res. Rep. no. 67-2369.

WANG, C. P. 1972 Appl. Phys. Lett. 20, 339.

Wells, C. S. (ed.) 1969 Viscous Drag Reduction. Plenum Press.

Whiте, A. 1969 In Viscous Drag Reduction (ed. C. S. Wells), pp. 297-311. Plenum Press.

WнIте, D. A. 1967 J. Fluid Mech. 28, 195.

YeH, Y. \& Cummings, H. 1964 Appl. Phys. Lett. 4, 176. 